\title{
Towards a 10-fs hyperspectral spectroscopy source with arbitrary pulse shaping based on adiabatic frequency conversion
}

\author{
Dylan A. Heberle*, Noah R. Flemens, Xiaoyue Ding, Wei-Zung Chang, and Jeffrey Moses \\ School of Applied and Engineering Physics, Cornell University, Ithaca, New York 14853, USA
}

\begin{abstract}
We introduce a 10-fs hyperspectral source architecture for facilitating nonlinear spectroscopy with multi-color sequences of arbitrarily shaped $10-\mathrm{fs}$ UV/Vis, near-IR, and mid-IR pulses. Design principles and initial experimental results are provided.
\end{abstract}

In the past decade, the nonlinear spectroscopy community has shown increased interest in ultrafast dynamics involving multiple degrees of freedom, which often correspond to distinct, well-separated areas of the electromagnetic spectrum. Methods in 2D vibrationalelectronic spectroscopy and pump-probe spectroscopy of rapidly changing electronic structure near a conical intersection following photoexcitation - for example, in rhodopsin, DNA, and electrocyclic ring opening reactions - have required combinations of ultrafast UV/Vis, near-IR, and/or mid-IR pulses [1-4]. Moreover, the dynamics of interest often occur in just tens of femtoseconds, requiring large absolute bandwidths at each spectral range. A 10-fs, energetic spectroscopy source capable of producing broadband, shaped and synchronized pulse pairs combining - as needed - UV/Vis, near-IR, and/or mid-IR portions of the electromagnetic spectrum would drive the field forward for such applications. Historically, high peak power and ultrafast spectroscopy sources have relied on Ti:sapphire amplifiers together with a combination of optical parametric amplifiers and sum- or difference-frequency generation stages. Though workhorses of the field, these systems have a number of limitations for these applications. 1) The limited average power of the amplifier means these systems are typically limited to few-kHz repetition rates that hinder the acquisition speed of a multidimensional experiment. 2) 10-fs duration pulses at mid-IR frequencies require greater than octave-spanning bandwidth, a great challenge to generate at suitably high energy. 3) The nonlinear stages generally do not preserve amplitude and phase profiles, requiring post-conversion pulse shaping, which is costly and difficult when managing dispersion at several frequency ranges simultaneously, even more so when greater-than-octave spanning bandwidths are involved.

In this work, we introduce a 10-fs nonlinear hyperspectral spectroscopy source architecture that overcomes the aforementioned constraints. The architecture consists of a near-IR optical parametric chirped pulse amplifier (OPCPA) pumped by a high average power picosecond amplifier, near-IR pulse shapers, and adiabatic frequency upconversion and downconversion stages. The adiabatic frequency conversion method was recently

* Corresponding author: dah378@,cornell.edu 
shown to allow arbitrarily shapeable microjoule pulses with single-cycle duration in the mid-IR and to allow the shift of an ultrabroad frequency band through linear spectral phase and amplitude transfer functions [5], thus greatly simplifying the technical requirements of arbitrary pulse shaping across a hyperspectral platform. The advancements in these technologies have coalesced to enable a uniquely suited, flexible spectroscopy source, allowing the combination and arbitrary shaping of microjoule-energy, 10-fs pulse sequences covering several octaves at UV/Vis, near-IR, and mid-IR wavelengths.

The design (Fig. 1) of our prototype system is as follows. We chose three distinct bands for simultaneous operation, at visible $(0.45-0.51 \mu \mathrm{m})$, mid-IR $(1.9-4.3 \mu \mathrm{m})$ and near-IR $(0.65-1.01-\mu \mathrm{m})$ frequency ranges, each with transform-limited duration $\leq 10 \mathrm{fs}$. We note that upconversion, downconversion, and OPCPA stages can be added to cover additional spectral ranges. The design employs a near-IR front end based on a high average power (200 W) 10-kHz Yb:YAG amplifier (Amphos) operating at $1030 \mathrm{~nm}$, an OPCPA, and dual near-IR pulse shapers. The Yb:YAG amplifier has two exit ports that share a common preamplifier, ensuring optical synchronization. From the first exit port, 900-fs pulses pump white light generation (WLG) in bulk YAG to generate a visible/near-IR continuum. The second exit port generates 3-ps pulses that are frequency doubled to pump a two-stage noncollinear OPCPA to generate amplified near-IR pulses with $200-\mu \mathrm{J}$ energy. Preconversion dispersion management and arbitrary pulse shaping is achieved with a pair of near-IR 4-f pulse shapers (PhaseTech). These shapers admit an optical bandwidth of 240 $\mathrm{nm}$ (tunable by choice of diffraction gratings) and have an update rate up to $25 \mathrm{kHz}$. The shaped near-IR pulses from the front end are then directed to the various adiabatic conversion stages, where they mix in aperiodically poled quasi-phase matching gratings with a strong (up to $1-\mathrm{mJ}$ energy) 1030-nm pump pulse from the $\mathrm{Yb}$ :YAG amplifier. Visible and mid-IR pulses are generated through adiabatic sum frequency generation (ASFG) in KTP (Raicol Crystals) and adiabatic difference frequency generation (ADFG) in lithium niobate (HC Photonics), respectively. Importantly, adiabatic frequency conversion enables a linear transfer of spectral amplitude through near-one-to-one photon number conversion - nearly every near-IR photon is converted to the targeted range - as well as a linear transfer of spectral phase [5]. Thus, our source architecture is designed to allow dispersion management and pulse shaping for each spectral range through a near-IR pulse shaper prior to the conversion stage, eliminating a need for separate post-conversion shaping devices for each spectral range. Moreover, the aperiodically poled adiabatic conversion gratings are designed to employ intrinsic pulse shaping - the use of wavelength dependent longitudinal conversion positions - to achieve simultaneous adiabatic phase matching and cancellation of the complex material group delay dispersion (GDD) through the device [6]. The result is a device with zero net GDD, eliminating the need for postconversion dispersion management.

Following implementation of the near-IR system, we tested the design of the adiabatic

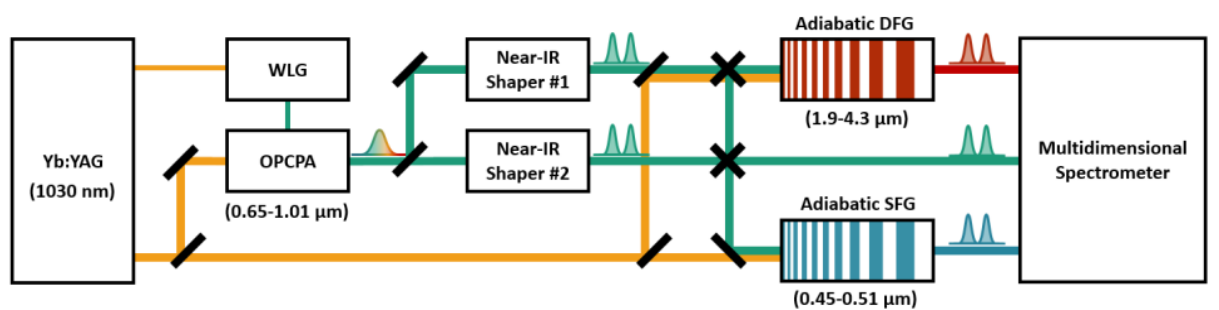

Fig. 1. Yb:YAG amplifier seeds WLG and pumps the OPCPA, ASFG, and ADFG stages. A portion of the WLG continuum $(0.65-1.01 \mu \mathrm{m})$ is amplified in a two-stage OPCPA to $200-\mu \mathrm{J}$ pulse energy. The pulses are shaped in the near-IR before upconversion and downconversion by ASFG and ADFG, respectively. Each of the two shaper outputs can be used for generation of shaped UV/vis, near-IR, or mid-IR pulses to create hyperspectral pulse sequences as needed for a particular experiment. 
conversion stages by measuring the fractional depletion of the near-IR power spectrum (experimental traces, Fig 2). Through each stage, a flat photon-number conversion ranging from $80-90 \%$ is observed over a bandwidth corresponding to a 10 -fs transform limited pulse (corresponding to one optical cycle in the mid-IR and $\sim 8$ cycles in the visible). The expected group delay of the zero-GDD ASFG device, calculated by split-step Fourier method pulse propagation simulation, is shown in Fig. 2(a). At present, our ADFG (midIR) device does not employ intrinsic pulse shaping and is based on the design in Krogen et al. [5], which generated single-cycle pulses and pulse pairs through dispersion management. However, a similar zero-GDD design for the ADFG crystal has an expected peak-to-valley group delay range of $\sim 10$ fs over the full octave-spanning mid-IR bandwidth.

We note several more advantages of our source architecture for hyperspectral and ultrafast nonlinear spectroscopy. With programmable pulse shaping entirely carried out within the fixed near-IR front end, the rapid control of pulse sequences afforded by pulseshaper assisted multidimensional spectroscopy in a "pump-probe" geometry [7], such as the use of phase-cycling, pi-shifting for background subtraction, and interferometrically stable control of pulse pair delay, can be extended over multiple octaves of bandwidth. The 200$\mathrm{W}$ average power of the pump laser allows operation at a $10-\mathrm{kHz}$ repetition rate while maintaining microjoule pulse energies, which enables fast acquisition of multidimensional spectra when combined with the rapid update rate of the pulse shapers and $10-\mathrm{kHz}$ acquisition rate of modern MCT array detectors. Finally, the use of orientation patterned semiconductors [8] as adiabatic conversion stages, currently in testing, could potentially extend the hyperspectral range to the molecular fingerprint region of the mid-IR.

a.

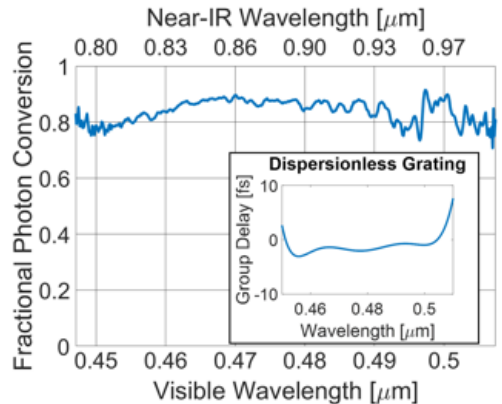

b.

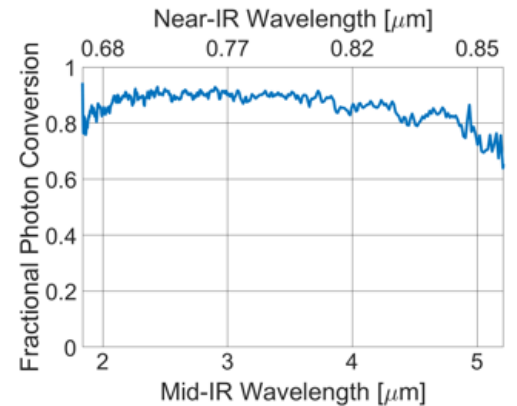

Fig. 2. Fractional photon conversion of adiabatic sum (a) and difference (b) frequency generation stages measured by the fractional depletion of the near-IR power spectrum. Inset shows simulated group delay of the ASFG crystal.

1. D. Polli, et al., Nature 467, 440 (2010)

2. W.J. Schreier, et al., Science 315, 625 (2007)

3. M.P. Minitti, et al., Phys. Rev. Lett. 114, 255501 (2015)

4. T.L. Courtney, et al., J. Chem. Phys. 143, 154201 (2015)

5. P. Krogen, et al., Nat. Photonics 11, 222 (2017)

6. N. Flemens, et al., "Single-Cycle or Arbitrarily Shaped Octave-Spanning Mid-Infrared Pulses: Intrinsic and Extrinsic Pulse Shaping in Adiabatic Frequency Conversion," in High-Brightness Sources and Light-driven Interactions, OSA Technical Digest (online) (Optical Society of America, 2018), paper MW2C.7

7. S.-H. Shim, M.T. Zanni, Phys. Chem. Chem. Phys. 11, 748 (2009)

8. P.G. Schunemann, "New Nonlinear Optical Crystals for the Mid-Infrared," in Advanced Solid State Lasers, OSA Technical Digest (online) (Optical Society of America, 2015), paper AM2A.2 